\title{
Unmasking the Masquerader: A Life Changing Therapeutic Trial
}

\author{
Bhanudeep Singanamalla ${ }^{1} \cdot$ Alisha Babbar $^{1} \cdot$ Phub Tenzin $^{1} \cdot$ Shivan Kesavan ${ }^{1} \cdot$ Priyanka Madaan $^{1,2} \cdot$ Lokesh Saini $^{1}$
}

Received: 3 March 2020 / Accepted: 30 March 2020 / Published online: 16 May 2020

(C) Dr. K C Chaudhuri Foundation 2020

To the Editor: A six-year-old boy, first born to a nonconsanguineous couple, presented with difficulty in walking and frequent falls since one and a half years of age (Supplementary video-1). The child also had history of frequent twisting postures of lower limbs initially, later involving upper limbs. These twisting postures subsided with sleep. On probing, the parents gave a history of diurnal variation such that the child used to be unable to walk unassisted by evening. There was no history of cognitive decline, behavioral issues, perinatal insult, and similar history in family. On examination, he had rigidity with dystonic posturing of both lower limbs. The gait was stiff with restricted arm-swing. He had brisk deep tendon reflexes, bilateral plantar flexor and striatal toe. In view of chronic progressive course, diurnal fluctuations in weakness and appendicular dystonia with normal cognition, a possible diagnosis of primary dystonia was kept. Magnetic resonance imaging (MRI) brain, serum ceruloplasmin, biotinidase assay, tandem mass spectrometry (TMS) and urine gas chromatography-mass spectrometry (GCMS) were normal. He was initiated on Syndopa trial and had a remarkable clinical response (Supplementary video-2). Clinical exome sequencing revealed an autosomal dominant, heterozygous, pathogenic missense variation (Chr.14: 55312502) in GCH1 gene.

Dopa responsive dystonia (DRD) was first recognized by Segawa et al. in 1976 but the term 'Dopa responsive dystonia' was suggested by Nygaard et al. in 1990 [1]. It is an autosomal dominant primary dystonia due to defect in guanosine triphosphate (GTP) cyclohydrolase I (GCH1 or DYT5). GTP cyclohydrolase I catalyses the rate limiting step in the production of

Electronic supplementary material The online version of this article (https://doi.org/10.1007/s12098-020-03281-4) contains supplementary material, which is available to authorized users.

Lokesh Saini

drlokeshsaini@gmail.com

1 Pediatric Neurology Division, Department of Pediatrics, Advanced Pediatric Centre, Post Graduate Institute of Medical Education and Research (PGIMER), Chandigarh, India

2 Council of Scientific and Industrial Research, New Delhi, India tetrahydrobiopterin (BH4), which has a crucial role in dopamine, folate and biopterin synthesis pathways [2]. Decrease in dopamine leads to the classical symptoms seen in DRD. Typical clinical features include childhood onset of limb dystonia, mainly in lower limbs that can progress to generalized dystonia and diurnal fluctuations in about $56-80 \%$ of the patients. The response to levodopa is excellent and sustained long-term without side-effects of those seen in adults. DRD is often misdiagnosed as dyskinetic or diplegic cerebral palsy [3]. In such cases, perinatal history, early markers of cerebral palsy, and neuroimaging may be helpful $[4,5]$. The index child highlights the often-seen delay in diagnosing DRD, and the importance of therapeutic trial of Syndopa in children with unexplained movement disorder, or spasticity.

Authors' Contribution BS and SK: involved in patient-care, did the literature review and prepared the initial draft of the manuscript; $\mathrm{AB}$ and PT: involved in patient-care and did the literature review; PM and LS: involved in patient-care, critically reviewed the manuscript and provided intellectual input. Dr. Naveen Sankhyan will act as Guarantor for this paper.

\section{Compliance with Ethical Standards}

Conflict of Interest None.

\section{References}

1. Segawa M, Hosaka A, Miyagawa F, et al. Hereditary progressive dystonia with marked diurnal fluctuation. Adv Neurol. 1976;14: 215-33.

2. Tadic V, Kasten M, Brüggemann N, Stiller S, Hagenah J, Klein C. Dopa-responsive dystonia revisited: diagnostic delay, residual signs, and nonmotor signs. Arch Neurol. 2012;69:1558-62.

3. Nygaard TG, Waran SP, Levine RA, Naini AB, Chutorian AM. Dopa-responsive dystonia simulating cerebral palsy. Pediatr Neurol. 1994;11:236-40.

4. Madaan P, Reddy C, Saini L. Gaze palsy: an important diagnostic clue. J Pediatr. 2019;212:236.

5. Singanamala B, Noolu R, Madaan P, Saini L. Rhythmic tongue thrusting: a useful clinical sign. Pediatr Neurol. 2020;102:81-2.

Publisher's Note Springer Nature remains neutral with regard to jurisdictional claims in published maps and institutional affiliations. 